\title{
Shunt-diode rectifier: a new scheme for efficient piezoelectric energy harvesting
}

\author{
Alexis Brenes', Elie Lefeuvre', Adrien Badel', Seonho Seok', Chan-Sei Yoo ${ }^{3}$ \\ ${ }^{1}$ Centre for Nanoscience and Nanotechnology, Univ. Paris Sud-CNRS, Université Paris-Saclay, France \\ ${ }^{2}$ SYMME, Université Savoie Mont-Blanc, France \\ ${ }^{3}$ Electronic Convergence Materials \& Device Research Center, Korea Electronics Technology Institute, Republic of Korea \\ Corresponding author : \\ Dr. Alexis Brenes, alexis.brenes@u-psud.fr \\ Tel.: +33169153464 \\ Fax.: +33169154050
}

\begin{abstract}
This paper highlights the interest of shunt-diode rectifiers to optimize the performances of piezoelectric vibration energy harvesters based on linear load adaptation. It provides, for the first time, a full analytical study of the input power and frequency bandwidth of such a system. We show that this architecture is well-suited for piezoelectric energy harvesting. In the presented approach, a so-called shunt diode is connected across the electrodes of the piezoelectric transducer, which makes the piezoelectric voltage unidirectional instead of alternating. Thus, DC-DC converters usually used for power optimization can be directly connected to the transducer. We show that weakly-coupled linear inertial piezoelectric energy harvesters generate higher power when connected to a shunt-diode rectifier than to a bridge rectifier. Moreover, the frequency bandwidth is enlarged by the use of the single-diode shunt rectifier, especially for strongly-coupled piezoelectric generators. The proposed architecture is also interesting for low piezoelectric voltages, at which voltage drops across the diodes used for AC-DC conversion critically degrade the power efficiency. Experimental results validate the presented analytical study and highlight the higher efficiency of the shunt-diode rectifier circuit. Compared to a standard bridge rectifier architecture, we demonstrate an increase of $+43 \%$ output power on a weakly-coupled generator and an increase of $+5 \%$ bandwidth on a strongly-coupled generator.
\end{abstract}

Keywords - Energy harvesting, piezoelectric devices, resonant power conversion, impedance matching.

\section{INTRODUCTION}

$\mathrm{M}$ ANY technological solutions have been developed to improve the performances of vibration energy harvesters. Among these, the use of piezoelectric transducers represents a viable approach considering the relatively high energy density obtained with such active materials [1]. The simplest piezoelectric energy harvesting architectures take advantage of half-bridge (HB) or full-bridge $(\mathrm{FB})$ diode rectifiers to ensure a one-directional charge flow transforming alternating piezoelectric voltage into DC voltages [2]. However, no existing architecture can reach the maximum available power independently of the mechanical vibration frequency and lots of works are still ongoing to increase the power harvested by piezoelectric generators [3, 4, 5]. Moreover, harvesting energy from the vibrations of the environment to power microsystems remains even a tougher challenge at low voltage amplitudes, where the imperfections of electronic components limit the overall performance, such as the voltage drop across the diodes under use [2].

To overcome this limitation, several works have focused on alternatives to standard HB or FB rectifiers [6, 7, 8, 9, 10]. For instance, voltage doubler topologies or resonant inductance-based rectifiers have been considered [11, 12]. Even for these circuits, theoretical predictions and experiments have proven that the forward voltage of on-chip diodes still has a strongly negative impact on the electrical power output [12]. The latter issue can be solved by the use of active rectifiers instead of diode-based rectifiers [13] or rectifier-free circuits [14] which increase the complexity of implementation, especially at ultra-low power.

In the field of microwave power harvesting techniques, shunt-diode rectifiers have been introduced in rectennas more than 15 years ago. These rectifiers were originally studied in 1990 and their very good power efficiency for rectification of voltage delivered by an ideal voltage source was well-known [15]. In 1998, McSpadden et al. used such a rectifier to achieve a high power conversion efficiency in microwave rectennas [16]. Such simple and efficient rectifiers are still widely used in microwave power conversion technologies [17, 18, 19] and/or inductive power transfer [20]. This rectifier circuit has also been used in specific synchronized-switching architectures for piezoelectric harvesters [21], sometimes by taking advantage of the body-diode of a MOSFET transistor, which plays the role of a rectifier [22]. Despite these few practical implementations in the field of piezoelectric energy harvesting, no analytical study has been performed, to date, to model and optimize the complex behavior of the electromechanical coupling in such systems when connected to a shunt-diode rectifier circuit.

In this work, we study the impact of a shunt-diode rectifier on the power response of a piezoelectric energy when connected to a DC-DC converter with impedance matching [3, 23, 24]. We determine the role of the resonator properties on the overall performance of this architecture. The results are compared with the classical case of resistive impedance matching through a HB and/or FB rectifier. 
This paper is organized as follows. In section II, we introduce the chosen architecture and the assumptions of our study and present the mathematical developments and results. A special focus is made on the impact of the electromechanical coupling and quality factor of the resonator on the piezoelectric output power. Experimental validations are reported in section III.

\section{STUDY OF THE PIEZOELECTRIC ENERGY HARVESTER WITH SHUNT-DIODE RECTIFIER}

This section presents a model predicting the power generated by a piezoelectric resonator when connected to a shunt-diode rectifier and a DC-DC converter. This extracted power will be called "input power" in the rest of the paper since it is the input power of the electrical circuit. We will show how the equivalent load resistance can be tuned to maximize this input power. Due to its similarities with the impedance matching notion of classical circuit theory, this situation is defined as "resistive impedance matching". Nevertheless, we are dealing with a nonlinear coupled system and one should keep in mind that this definition corresponds to the extension of impedance matching to nonlinear position-dependent circuits $[25,3,26,27]$ and not to the initial definition of classical circuit theory.

\section{A. General description of the system under study}

The electromechanical transducer is considered here as a linear single degree of freedom (SDOF) piezoelectric resonator. Despite well-known limitations in large amplitude regimes [28, 29], this linear model broadly used in the literature [4, 30] often provides a good behavioral description for numerous structures around one of their resonant frequency. The validity of this model has been confirmed in our case (see section III).

This lumped SDOF model is composed of an inertial mass $M$ suspended by a spring of stiffness $K$ (Figure 1). The natural angular frequency in the open-circuit configuration is $\omega_{0}=\sqrt{K / M}$. The motion $y$ of the resonator case gives rise to an internal displacement $x$ of the inertial mass with respect to the case. The harmonic acceleration of the base is written $\frac{d^{2} y}{d t^{2}}=-\gamma \sin (\omega t+\phi)$. The damper $c$ models the mechanical losses of the system. The factor $\alpha\left(\mathrm{N} \mathrm{V}^{-1}\right)$ accounts for the bi-directional coupling between the mechanical and the electrical domains. The capacitor $C_{p}$ represents the internal capacitance of the piezoelectric element. For the sake of readability, some variables are normalized, as reported in Table I. Figure 1 depicts the typical structure of the considered power conversion circuit, composed of a rectifier, a DC-DC converter enabling to maximize the power extracted from the electromechanical resonator (resistive impedance matching) and an energy storage unit (e.g. capacitor, supercapacitor or battery) whose voltage is written $u_{D C}$. The power consumed by the load powered by the energy harvesting system is modeled by the resistor $R_{\text {load }}$.

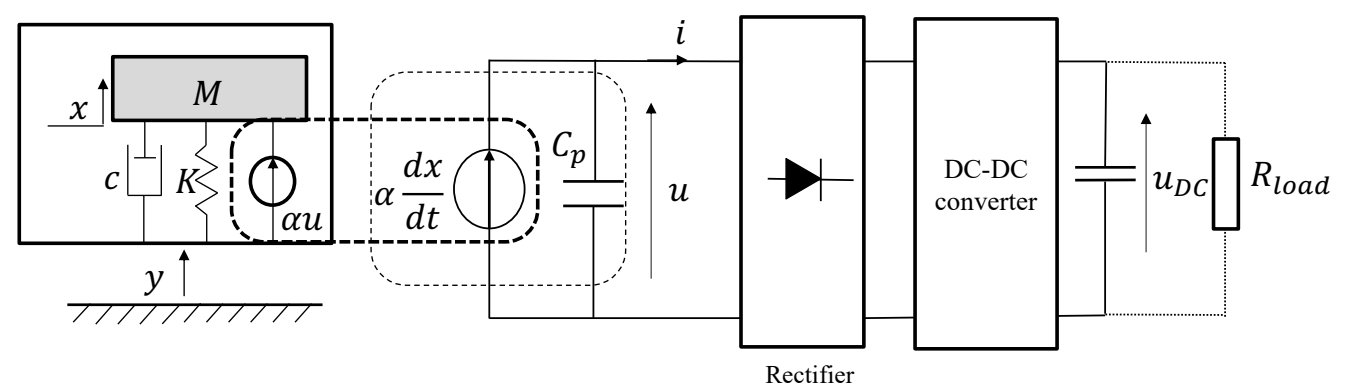

Figure 1: SDOF linear model of inertial piezoelectric energy harvesters and typical structure of the interface circuit.

\section{B. Shunt-diode rectifier architecture}

The proposed architecture is depicted in Figure 2. It is composed of the electromechanical resonator, a shunt-diode rectifier and a buck-boost DC-DC converter [31].

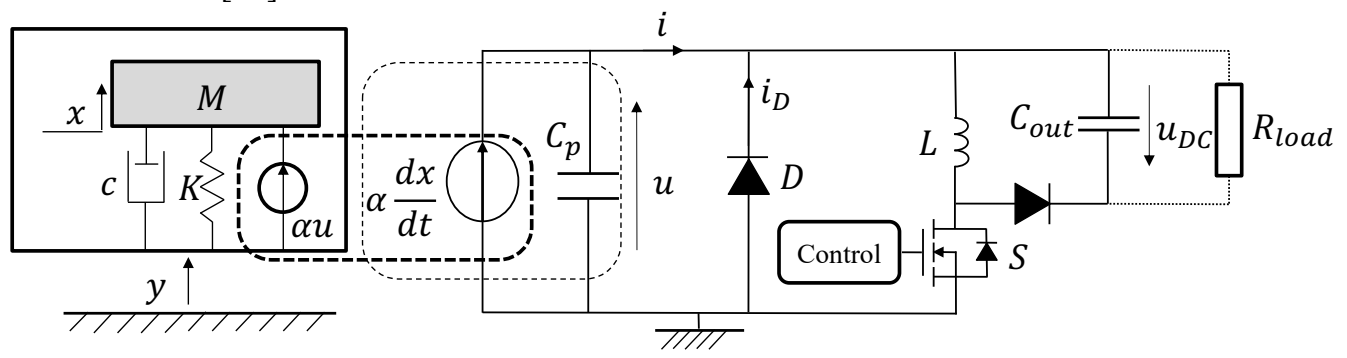

Figure 2: Proposed circuit with shunt-diode rectifier and DC-DC buck-boost converter.

At first sight, this architecture has some similarities with the architecture given in [32], which proposes a circuit with a FB rectifier and no smoothing capacitor. However, the behavior of our system is different due to the electromechanical coupling and the nonlinear relationship between the voltage and displacement of the resonator. The theoretical waveforms of the displacement $x$, the piezoelectric voltage $u$ and current $i$, as well as the shunt-diode current $i_{D}$ are depicted in Figure 3 . 
The displacement of the resonator is assumed to remain sinusoidal despite the non-sinusoidal shape of $u$ (first-harmonic approximation), which is a common assumption for high-Q factor resonators [30]. Due to the shunt diode, the piezoelectric voltage is always positive. The diode $D$ (see Figure 2) conducts each time the piezoelectric voltage is null. The buck-boost converter operates in discontinuous conduction mode. Its input current is reflected by the positive peaks of the piezoelectric current.
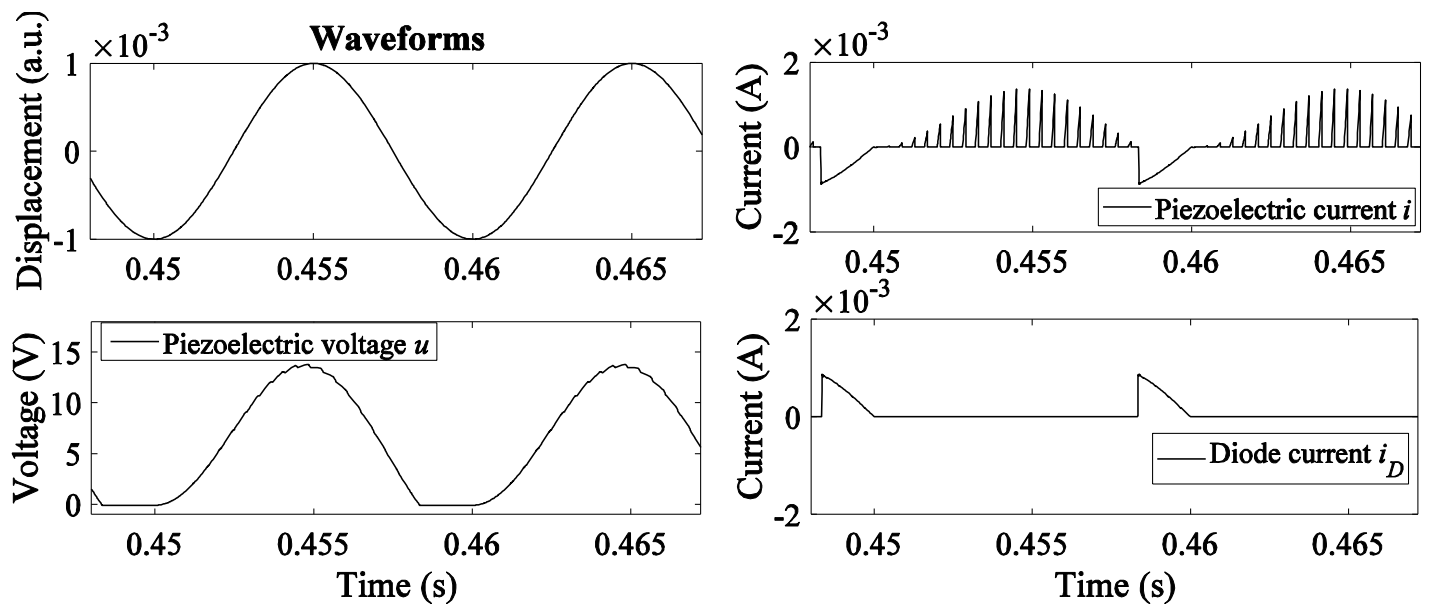

Figure 3: Illustration of the theoretical displacement, voltage and current waveforms in the shunt-rectifier architecture.

\section{Analytical model of the proposed architecture}

With respect to the resonator, the DC-DC converter exhibits an input resistance $R$. This input resistance is determined by the value of inductor $L$, the duty-cycle $\delta$ and the switching frequency $f_{s}$ of the electronic switch $S$. In discontinuous conduction mode, it is written (1) as long as the switching frequency is very large compared to the frequency of the mechanical oscillation, i.e. $f_{S} \gg$ $\omega / 2 \pi[33,34]$.

$$
R=\frac{2 L f_{s}}{\delta^{2}}
$$

We focus here on the case where the impact of the threshold voltage $u_{D}$ of diode $D$ can be neglected (i.e. the acceleration is sufficient to ensure $u_{D} \ll \max (u)$ ). As long as the voltage $u$ is positive, all the piezoelectric current flows through the buck-boost converter. When the voltage $u$ reaches zero, the current flows directly from the piezoelectric generator through the diode $D$. Therefore, the equations governing the motion of the resonator and its output voltage are given by (2).

$$
\left\{\begin{array}{c}
M \frac{d^{2} x}{d t^{2}}+c \frac{d x}{d t}+K x+\alpha u=M \gamma \sin (\omega t+\phi) \\
\forall t \in\left[\frac{2 n \pi}{\omega}, t_{1}+\frac{2 n \pi}{\omega}\right], \frac{u}{R}=\alpha \frac{d x}{d t}-C_{p} \frac{d u}{d t} \\
\forall t \in\left[t_{1}+\frac{2 n \pi}{\omega}, \frac{2(n+1) \pi}{\omega}\right], u=0 \text { and } i=\alpha \frac{d x}{d t}
\end{array}\right.
$$

In equations (2), $t_{1}$ is the instant at which $u$ reaches zero. Considering the normalization given in Table I and defining the angle $\theta$ by $\theta=\omega_{0} t$, the system (2) becomes (3).

$$
\left\{\begin{array}{c}
\frac{d^{2} X}{d \theta^{2}}+\frac{1}{Q} \frac{d X}{d \theta}+x+\frac{u}{u_{0}}=\sin (\Omega \theta+\phi) \\
\forall \theta \in\left[\frac{2 n \pi}{\Omega}, \theta_{1}+\frac{2 n \pi}{\Omega}\right], u(\theta)=r u_{0}\left[k_{m}^{2} \frac{d X}{d \theta}-\frac{1}{u_{0}} \frac{d u}{d \theta}\right] \\
\forall \theta \in\left[\theta_{1}+\frac{2 n \pi}{\Omega}, \frac{2(n+1) \pi}{\Omega}\right], u=0 \text { and } i=i_{0} k_{m}^{2} \frac{d X}{d \theta}
\end{array}\right.
$$

where $n$ is an integer, $Q=M \omega_{0} / c$ is the quality factor of the resonator, $r=R C_{p} \omega_{0}$ is the normalized input resistance of the DCDC converter, $k_{m}^{2}=\alpha^{2} / K C_{p}$ is the modified electromechanical coupling coefficient, $u_{0}=M \gamma / \alpha, i_{0}=C_{p} M \omega_{0} \gamma / \alpha$ and $\theta_{1}=\omega_{0} t_{1}$. For the analysis, we also define the electromechanical coupling coefficient as $k^{2}=$ $\alpha^{2} /\left(K C_{p}+\alpha^{2}\right)=k_{m}^{2} /\left(1+k_{m}^{2}\right)$. 
Due to the electromechanical coupling, the motion of the resonator $X=X_{m} \sin (\Omega \theta)$ is influenced by the piezoelectric voltage. The analytical expression (4) of the voltage was derived from (3) in the time domain, for $\theta \in[0,2 \pi / \Omega]$.

$$
\begin{aligned}
& \text { TABLE I } \\
& \text { VARIABLES AND NORMALIZATION } \\
& \forall \theta \in\left[0, \theta_{1}\right], u(\theta)=\frac{u_{0} e^{-\frac{\theta}{r}}}{1+r^{2} \Omega^{2}}\left[X_{m} k_{m}^{2} r^{2} \Omega^{2}\left(1+\frac{e^{\frac{\theta}{r}} \sin (\Omega \theta)}{r \Omega}-e^{\frac{\theta}{r}} \cos (\Omega \theta)\right)\right] \\
& \forall \theta \in\left[\theta_{1}, \frac{2 \pi}{\Omega}\right], u(\theta)=0
\end{aligned}
$$

\begin{tabular}{ccc}
\hline \hline Variable & Quantity (unit) & Normalized variable \\
\hline$\omega$ & Vibration angular frequency (rad. s $\left.{ }^{-1}\right)$ & $\Omega=\omega / \omega_{0}$ \\
$y$ & Displacement of the base (m) & $Y=y \omega_{0}^{2} / \gamma$ \\
$x$ & Displacement of the resonator (m) & $X=x \omega_{0}^{2} / \gamma$ \\
$p$ & Piezoelectric output power (W) & $P=\frac{p}{p_{\text {lim }}} Q=\frac{8 p \omega_{0}}{M \gamma^{2}}$ \\
\hline \hline
\end{tabular}

One can note that no simple analytical expression of $\theta_{1}$ can be derived from (4) but this angle can be computed numerically by finding the first instant where $u$ cancels out. As an illustration, we report, in (5) an empirical law based on numerical computations enabling to predict $\theta_{1}$ for $V_{D}=0 \mathrm{~V}$, where $A_{1} \simeq 3.139, A_{0} \simeq 2.141$ and $B_{0} \simeq 0.337$. Depending on the desired precision, the degree of the numerator and denominator respect to $(r \Omega)^{-1}$ can be increased, if required.

$$
\theta_{1}=\frac{1}{\Omega} \frac{A_{1}(r \Omega)^{-1}+A_{0}}{(r \Omega)^{-1}+B_{0}}
$$

The corresponding fit is reported in Figure 4. One can notice the very good agreement between the empirical law (5) and the numerical computations of $\theta_{1}$, the error being smaller than $0.3 \%$ on the considered range of $r \Omega$.

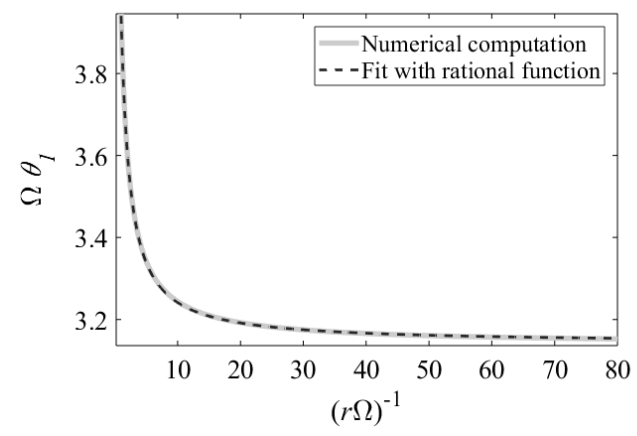

Figure 4: Numerical computation of $\Omega \theta_{1}$ as a function of $(r \Omega)^{-1}$ and fit with rational function.

As will be shown in subsection $\mathrm{D}$, the expression (4) of $u$ is critical for the determination of the power harvested by the system.

D. Estimation of the power response and comparison with other classical bridge-rectifier architectures

Starting from (4), the method of harmonic balance [26, 27] yields the system of equations (6).

$$
\left\{\begin{array}{c}
\int_{0}^{2 \pi}\left[X_{m} \sin \varphi-X_{m} \Omega^{2} \sin \varphi+\frac{u(\varphi)}{u_{0}}\right] \sin \varphi d \varphi=\int_{0}^{2 \pi}[\sin (\varphi+\phi)] \sin \varphi d \varphi \\
\int_{0}^{2 \pi}\left[\frac{X_{m} \Omega \cos \varphi}{Q}+\frac{u(\varphi)}{u_{0}}\right] \cos \varphi d \varphi=\int_{0}^{2 \pi}[\sin (\varphi+\phi)] \cos \varphi d \varphi
\end{array}\right.
$$

From (6), the expression (7) of the normalized amplitude $X_{m}$ of the motion, is obtained

$$
X_{m}=\frac{1}{\sqrt{\left(1-\Omega^{2}+k_{m}^{2} U_{p}\right)^{2}+\left(\frac{\Omega}{Q}+k_{m}^{2} U_{q}\right)^{2}}}
$$


where $U_{p}$ and $U_{q}$ relate to the in-phase and quadrature components (with respect to $x$ ) of the voltage $u$ and are defined by (8).

$$
\left\{\begin{array}{l}
U_{p}=\frac{1}{\pi k_{m}^{2} X_{m} u_{0}} \int_{0}^{2 \pi} u(\varphi) \sin \varphi d \varphi \\
U_{q}=\frac{1}{\pi k_{m}^{2} X_{m} u_{0}} \int_{0}^{2 \pi} u(\varphi) \cos \varphi d \varphi
\end{array}\right.
$$

The detailed expressions of $U_{p}$ and $U_{q}$ are given in (9).

$$
\left\{\begin{array}{c}
U_{p}=\frac{r^{2} \Omega^{2}}{2 \pi\left(1+r^{2} \Omega^{2}\right)}\left[\Omega \theta_{1}+\frac{\sin \left(2 \Omega \theta_{1}\right)}{2}-\frac{\sin ^{2}\left(\Omega \theta_{1}\right)}{r \Omega}-\frac{2 e^{-\frac{\theta_{1}}{r} r} \Omega\left(e^{\frac{\theta_{1}}{r}}-\cos \left(\Omega \theta_{1}\right)+r \Omega \sin \left(\Omega \theta_{1}\right)\right)}{1+r^{2} \Omega^{2}}\right] \\
U_{q}=\frac{r \Omega}{2 \pi\left(1+r^{2} \Omega^{2}\right)}\left[\Omega \theta_{1}-\frac{\sin \left(2 \Omega \theta_{1}\right)}{2}-r \Omega \sin ^{2}\left(\Omega \theta_{1}\right)+\frac{2 e^{-\frac{\theta_{1}}{r} r^{2} \Omega^{2}\left(e^{\frac{\theta_{1}}{r}} r \Omega-\sin \left(\Omega \theta_{1}\right)-r \Omega \cos \left(\Omega \theta_{1}\right)\right)}}{1+r^{2} \Omega^{2}}\right]
\end{array}\right.
$$

From (7), the expression (10) of the normalized input power is finally obtained.

$$
\begin{gathered}
P=\frac{p}{p_{\text {lim }}} Q=\frac{\Omega^{2} k_{m}^{2} r X_{m}^{2}}{\pi\left(1+r^{2} \Omega^{2}\right)^{2}}\left[2\left(\Omega \theta_{1}-r \Omega+\left(1-e^{-\frac{2 \theta_{1}}{r}}\right) r^{3} \Omega^{3}+r^{2} \theta_{1} \Omega^{3}\right)-\sin \left(2 \Omega \theta_{1}\right)\right. \\
\left.+r \Omega\left(2 \cos \left(2 \Omega \theta_{1}\right)+r \Omega\left(\sin \left(2 \Omega \theta_{1}\right)-8 e^{-\frac{\theta_{1}}{r}} \sin \left(\Omega \theta_{1}\right)\right)\right)\right]
\end{gathered}
$$

where $p_{\text {lim }}=\frac{M \gamma^{2} Q}{8 \omega_{0}}$ is the maximum power that can be extracted from the generator [3, 26].

From (10), one can deduce, for each normalized frequency $\Omega$, the optimal value $r_{o p t}(\Omega)$ of the normalized input resistance (corresponding to an input resistance $R_{\text {opt }}$ ) for which $P(\Omega)$ is maximized, and then infer from (1) the value of the corresponding optimal duty-cycle $\delta$ to apply to the DC-DC converter. This may be done numerically by solving the nonlinear equation $\frac{d P}{d r}=0$ with respect to $r$. However, we do not advise to choose this method. Indeed, it requires to start from (10), then replace $X_{m}$ by its expression as a function of $\theta_{1}$ (with (7) and (9)), then replace $\theta_{1}$ by an empirical law as a function of $r$ (like (5)) and derive the obtained expression with respect to $r$. The resulting equation takes pages to write down and must then be solved numerically, which is extremely time-consuming because of its complexity. In our experience, it is much more reasonable to try, for each frequency $\Omega$, a set of values of $r$ for which $P$ is computed from (10) and then choose the one that maximizes $P$. This is what we have done here. The result is plotted in Figure 5 for several values of the product $k^{2} Q$, which is the common figure of merit for piezoelectric generators excited monochromatically $[3,26,35]$.

In Figure 5, we plot and compare the results with those of a HB rectifier circuit with smoothing capacitance (see Figure 6). One can see that, for high values of $k^{2} Q$, the optimal power $P_{\text {opt }}$ exhibits two maxima. The power responses of the FB rectifier circuit are not reported here because they are almost identical to those of the HB rectifier circuit, the main difference lying in the value of the optimal input impedance of the DC-DC converter.

One can notice that the power responses obtained with the proposed method are not flat, i.e. one can only reach the maximum power for one or two frequencies. This is because our control scheme has a single adjustable parameter (the equivalent input impedance of the DC-DC converter). To reach a plateau of input power, one should manage to tune separately the electrical damping and the resonance frequency. This is impossible with the single adjustable parameter that we have here. To our knowledge, according to the state of the art in piezoelectric energy harvesting, the only way to tune separately the electrical damping and the resonance frequency is to opt for synchronous architectures with intelligent control which can introduce an adjustable phase shift between the piezoelectric current and the piezoelectric voltage.

As in the case of resistive impedance matching through a FB rectifier and smoothing capacitor [3], we are not aware of a simple analytical criterion to determine the existence of a pair of optimal resistances for which the input power is maximized. However, numerical simulations have led to the rule of thumb (11) for the appearance of two local maxima on the power response corresponding to a minimum and a maximum value of the matched normalized input resistor $r_{\text {opt }}$.

$$
k^{2} Q>3.4 \pi / 4
$$

For a deeper understanding, we write $P_{\max }\left(k^{2} Q\right)$ the maximum of $P_{\text {opt }}(\Omega)$ with respect to $\Omega$ and report, in Figure 7, the evolution of $P_{\max }$ as a function of $k^{2} Q$. We compare this evolution to the theoretical case of AC-load [33] (i.e. when the piezoelectric 
generator is connected to a single matched resistor) and to the case of a HB or FB rectifier bridge with smoothing capacitor (studied in $[3,10])$. One can notice that, in terms of electrical input power, the shunt-diode case is closer to the theoretical optimum.

Figure 5 and Figure 7 highlight that, as long as criterion (11) is not met, the power harvested by the shunt-diode circuit at resonance is larger than the power harvested by a bridge rectifier with smoothing capacitor. For strongly-coupled resonators, the same maximum power is achieved for all the rectifier circuits but the bandwidth of the shunt-diode system is larger than that of the bridge-rectifier system.
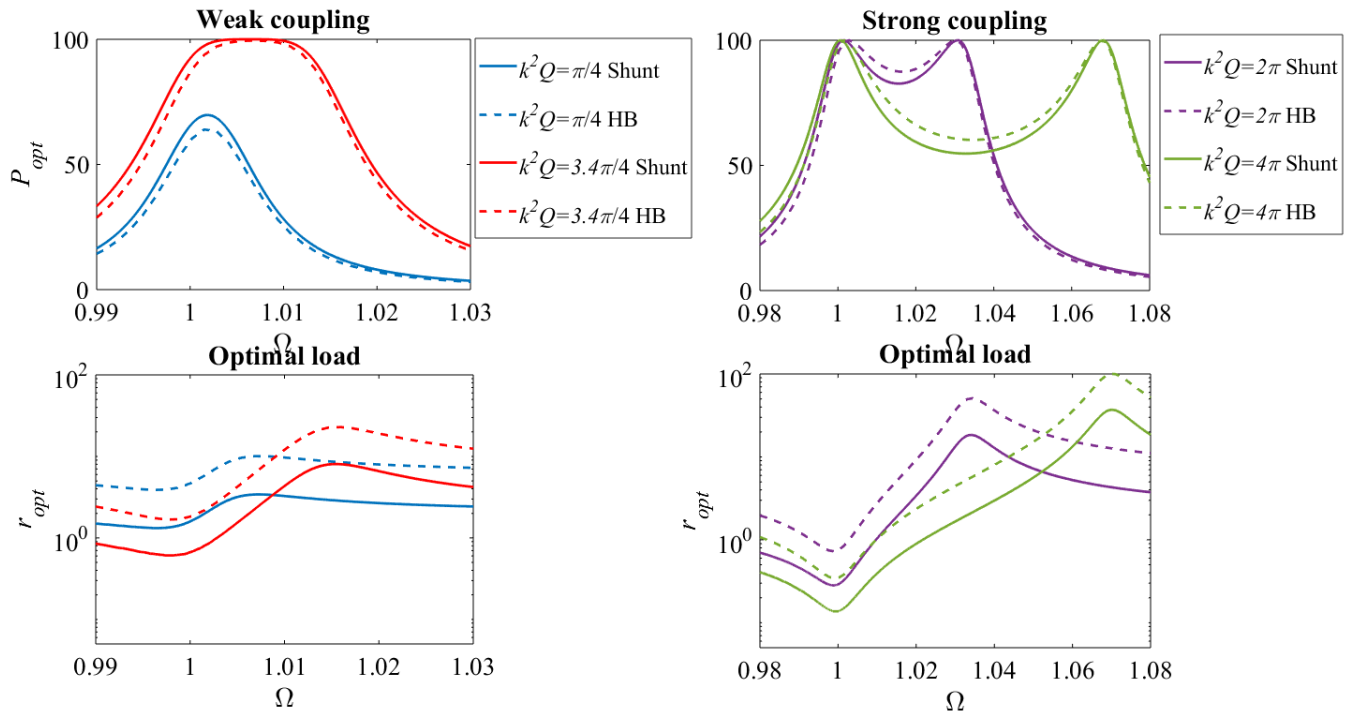

Figure 5: Normalized input power for resistive impedance matching with the shunt-diode rectifier (solid lines) and a classical resistive impedance matching on a HB rectifier with smoothing capacitor (dashed lines).

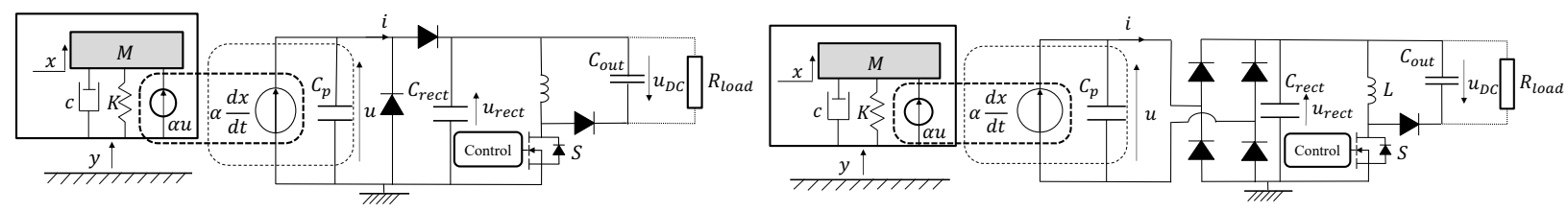

Figure 6: Upper: Architecture with HB rectifier and buck-boost converter. Lower: Architecture with FB rectifier and buck-boost converter.
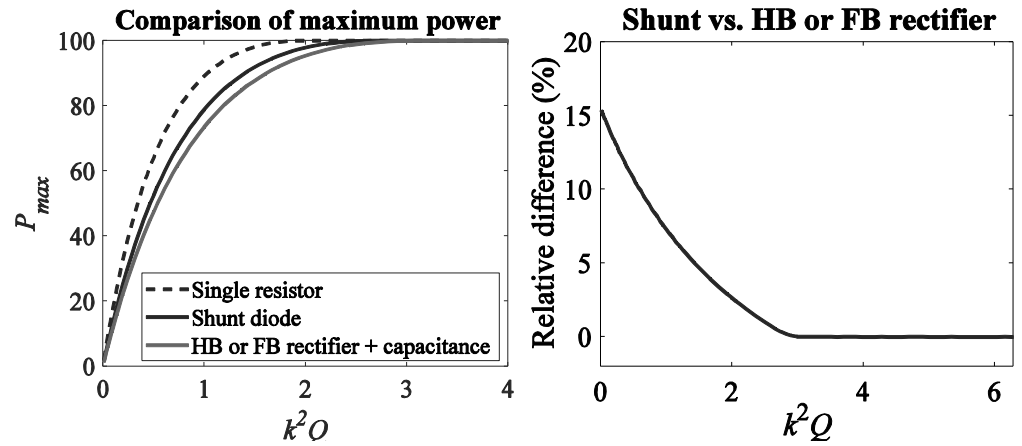

Figure 7: Left : Maximum input power vs. figure of merit $k^{2} Q$. Right : Comparison between the maximum power harvested by the shunt rectifier and by both bridge rectifier architectures.

\section{E. Comparison with synchronized-switching architectures}

Other architectures based on synchronized switching have been considered in the literature in order to increase the power harvested by the piezoelectric energy harvester. Amongst this category, series- and parallel-SSHI [12, 36, 37, 38] are especially efficient for weakly-coupled generators. The principle lies in the addition of a bias-flip stage made of an inductor and a switch. Their architectures are reported in Figure 8. The power vs. frequency responses of those SSHI architectures under the assumption of resistive impedance matching and single-harmonic behavior of the mechanical structure are given in Figure 9 [39].

In terms of optimal input power, Figure 9 shows that parallel- and series-SSHI are expected to improve the performance of weakly-coupled energy harvesters (the maximum power and the bandwidth being larger). As the coupling increases, the SSHI 
architectures become less and less interesting because their bandwidth does not increase. Simulations show that, as soon as $k^{2} Q \geq$ $3 \pi / 4$, the bandwidth of SSHI architectures becomes narrower than the bandwidth of the shunt-diode architecture.

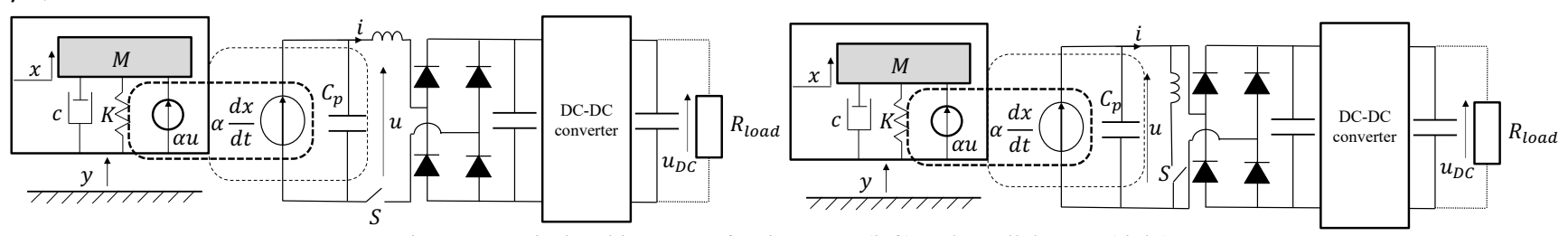

Figure 8: Typical architectures of series-SSHI (left) and parallel-SSHI (right).
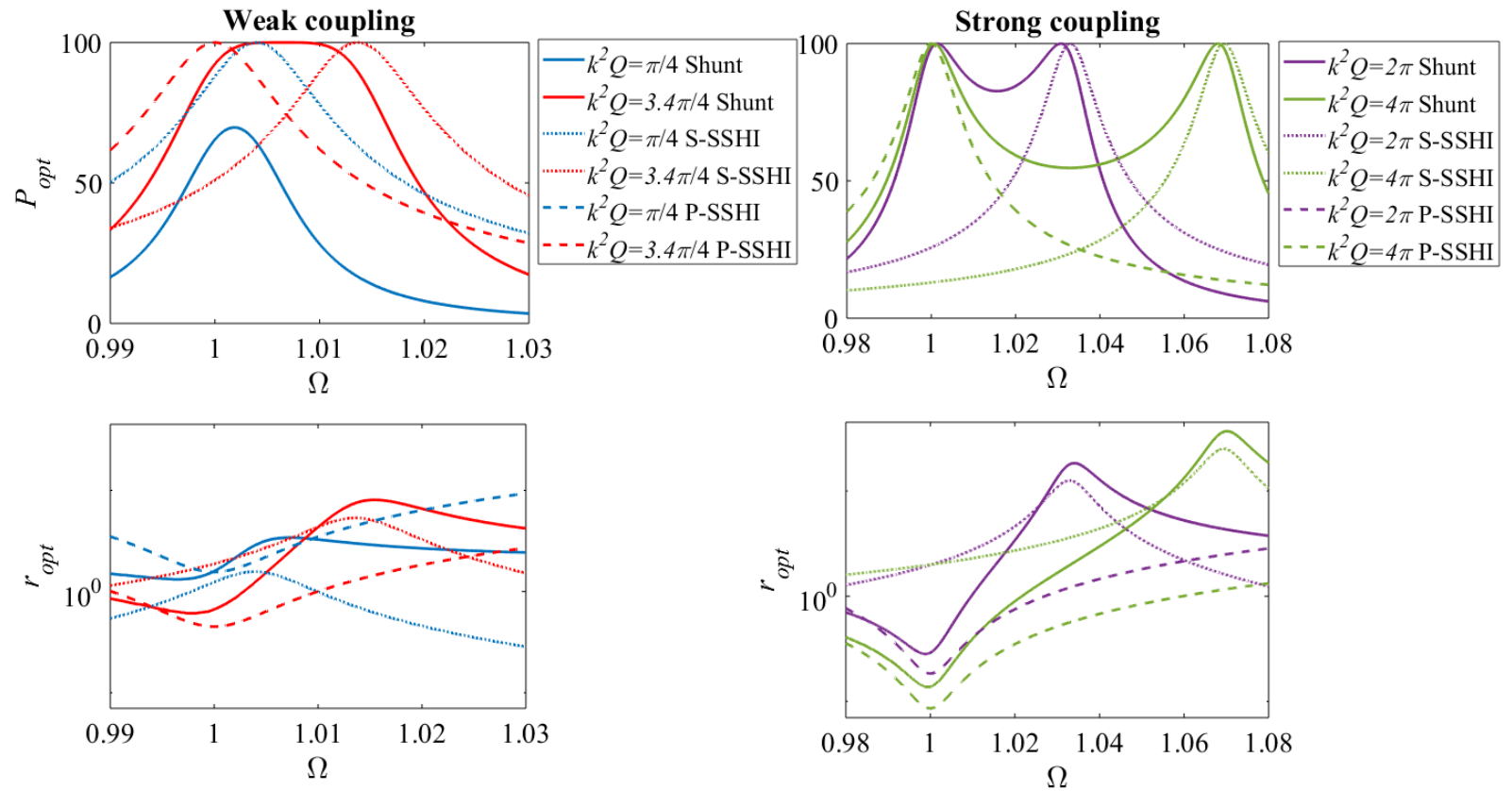

Figure 9: Normalized input power for resistive impedance matching and corresponding optimal impedance with the shunt-diode rectifier (solid lines), series SSHI (S-SSHI, dotted lines) and parallel SSHI (P-SSHI, dashed lines). The power vs. frequency responses of P-SSHI are superimposed.

\section{F. Interest and drawbacks of synchronized switching architectures}

Amongst the studied SSHI architectures (see section II.E), some expectedly bring out more input power than the shunt-diode architecture in the case of a weakly-coupled generator $\left(k^{2} Q \leq 3 \pi / 4\right)$. This advantage is combined with the expected improvement of the power efficiency brought by the increase of the voltage across the generator.

However, SSHI methods generate upper harmonics on the piezoelectric voltage which in turn tend to actuate higher mechanical modes of the resonator $[36,40]$. These parasitic modes reduce the accuracy of the switching instants, which degrades the overall performance especially for series-SSHI [41]. As a consequence, the effective implementation of such techniques requires a specific effort on the design of the mechanical structure and circuit (e.g. large inductance) to limit upper harmonics resonances. It can be noted that the proposed method generates harmonics as well, but at a very high frequency range and therefore with limited response of the structure.

Moreover, SSHI architectures request a bias-flip stage in addition to the rectifier and DC-DC converter stages (see Figure 8). This additional stage also introduces power losses. Last but not least, including this additional bias-flip stage requires the optimization of two control signals: the synchronous control signal of the bias-flip rectifier and the control signal of the DC-DC converter. Without the optimization of both control signals, the power vs. frequency responses of Figure 9 remain theoretical.

Notwithstanding these drawbacks, SSHI remains an interesting and efficient solution for piezoelectric energy harvesting but one should keep in mind that the choice of an optimal architecture results from a balance between all the design constraints (theoretical input power, power efficiency, consumption of the control scheme, complexity of the mechanical design and complexity of the electrical design...). All aspects should be balanced before choosing an appropriate solution. Any design choice between the shunt-diode architecture and synchronized switching relies more on a case-by-case optimization (average output power, coupling coefficient) than on a general statement which would remain valid for any piezoelectric generator.

The results of section II prove that the shunt-diode architecture exhibits interesting performances in comparison to other classical bridge-rectifier architectures and synchronized-switching architectures. In particular, we have proven that, in terms of input power, the performance of the shunt-diode rectifier is better than the other standard bridge rectifier circuits. Section III reports experimental evidence of our statements, at high and low voltages. 


\section{EXPERIMENTAL VALIDATION}

\section{A. Experimental setup}

The experimental validation is performed on two different piezoelectric generators composed of a $36 \times 36 \times 0.2 \mathrm{~mm}^{3} \mathrm{PZT}-5 \mathrm{H}$ plate bonded on a $60 \times 37 \times 0.5 \mathrm{~mm}^{3}$ stainless steel plate (see Figure 10), with a small seismic mass bonded close to the tip. The composite plate is clamped on a $40 \mathrm{~mm}$-long edge to a rigid aluminum base. A LDS V406 shaker drives this base through a LDS PA500L power amplifier. The acceleration of the base is measured using an accelerometer (B\&K DeltaTron 4517).

The rectifiers (see Figure 10) are composed of Schottky diodes (BAT46), with a very low threshold voltage (typically lower than $0.3 \mathrm{~V}$ ). A signal generator (Keysight $33600 \mathrm{~A}$ ) is used to drive the shaker's power amplifier with a sinusoidal signal. The amplitude of the sine wave is set by an automated feedback control loop to get the desired acceleration at the base of the device.

TABLE II

IDENTIFIED ELECTROMECHANICAL PARAMETERS

\begin{tabular}{ccc}
\hline \hline \multirow{2}{*}{ Parameter } & $\begin{array}{c}\text { Weakly-coupled resonator } \\
\text { Estimated value }\end{array}$ & $\begin{array}{c}\text { Strongly-coupled resonator } \\
\text { Estimated value }\end{array}$ \\
\hline$C_{p}$ & $186 \mathrm{nF}$ & $91 \mathrm{nF}$ \\
$f_{0}$ & $93.46 \mathrm{~Hz}$ & $115.95 \mathrm{~Hz}$ \\
$M$ & $7.1 \mathrm{~g}$ & $6 \mathrm{~g}$ \\
$\alpha$ & $2 \times 10^{-3} \mathrm{~N} \mathrm{~V}^{-1}$ & $3.5 \times 10^{-3} \mathrm{~N} \mathrm{~V}^{-1}$ \\
$k^{2}$ & $8 \times 10^{-3}$ & $4.5 \times 10^{-2}$ \\
$Q$ & 45 & 80 \\
$k^{2} Q$ & 0.36 & 3.6 \\
\hline
\end{tabular}
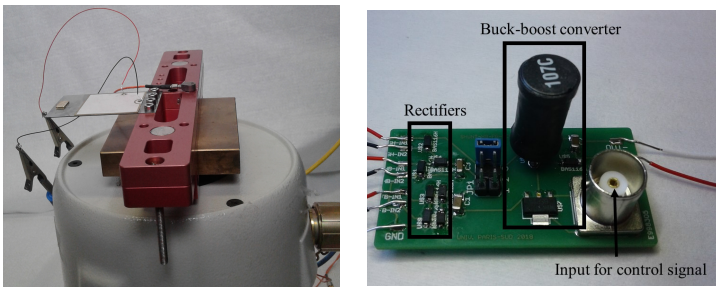

Figure 10: Pictures of the experimental setup. Left: Piezoelectric generator and shaker. Right: Electronic circuit.

The switching frequency of the DC-DC converter is set at $f_{s}=10 \mathrm{kHz}$. Its inductor is $100 \mathrm{mH}$ with less than $5 \Omega$ internal series resistance. The buck-boost converter (see Figure 2) is operated in discontinuous conduction mode. Since the control of the buckboost converter is operated externally, the consumption of the control circuit is not taken into account in our experiments. Compared to the power levels achieved here (hundreds of microwatts to several milliwatts), the power consumption of such a control stage would expectedly be very small (a few microwatts).

All the smoothing capacitors have $4.7 \mu \mathrm{F}$ capacitance and the output of the buck-boost converter is connected to a $R_{\text {load }}=50 \mathrm{k} \Omega$ resistive load. The corresponding input impedance of the buck-boost converter is then computed from (1). At each frequency, the duty-cycle is adjusted via an automatic procedure to reach the maximum input power. The algorithm computes the theoretical value of the optimal duty-cycle based on the resonator parameters and progressively reaches this duty-cycle with a PI controller. Then, finer adjustments are made step-by-step around that point to maximize the power. All signals are monitored using a digital oscilloscope (Agilent DSO-X 3014A), and the input and output powers are measured with a YOKOGAWA PX8000 powerscope.

The parameters of the resonator have been estimated from the measurements of the presented power response combined with capacitance measurements. A fit of admittance measurements is given in Figure 11 to illustrate the linear behavior of our resonator. These parameters are reported in Table II. The weakly-coupled resonator exhibits $k^{2} Q \simeq 0.36 \simeq 0.11 \pi$ so that the power harvested by the shunt rectifier is expected to be larger than the power harvested by the two other circuits (see section II). For the stronglycoupled resonator, $k^{2} Q=3.6$, which corresponds to a case where the difference in terms of bandwidth between the architectures should be large enough to be observed experimentally. As written in section II.C, there is no chance to observe a significant difference between the HB and FB rectifier circuits at such level (except that the optimal input impedance of the DC-DC converter would be different). Hence, in this section, we did perform measurements with only one of them (the HB rectifier).

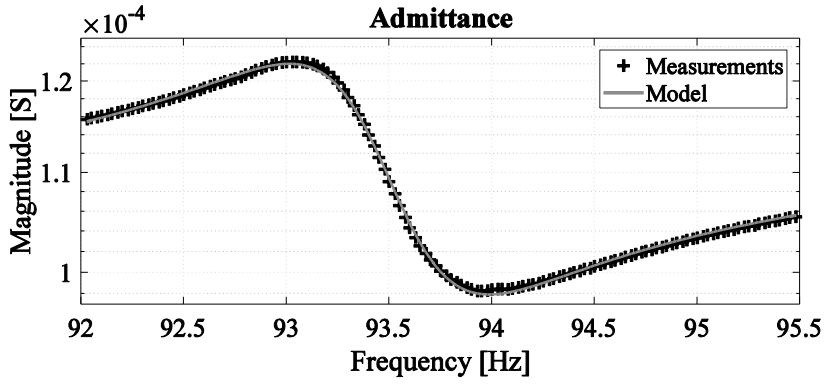

Figure 11: Admittance measurements around the resonance frequency and fit with linear SDOF model 


\section{B. Model validation and advantage of the shunt rectifier at high voltage}

For this first experiment, the acceleration amplitude is set so that the measured open-circuit voltage $u_{O C}$ at resonance (given by (12) [3]) is $25 \mathrm{~V}$. This corresponds to an acceleration $\gamma \simeq 17.7 \mathrm{~m} . \mathrm{s}^{-2}$ for the weakly-coupled generator, and $\gamma \simeq 4.05 \mathrm{~m}$. $\mathrm{s}^{-2}$ for the strongly-coupled generator. Comparing the architectures at similar voltage levels is useful to estimate the impact of the buckboost converter on the overall power efficiency. In this high-amplitude regime, the diode threshold voltage may be neglected, as will be proven by the comparison between our model and the experimental results.

$$
u_{O C}=\frac{k_{m}^{2} Q M \gamma}{\alpha}
$$

Typical waveforms obtained with the HB and shunt rectifiers are reported in Figure 12. In both situations, the duty-cycle has been adapted to reach the maximum power point at each frequency. In the case of a shunt-diode rectifier, one can identify the exponential start and the distorted sine-wave voltage shape, as described in Figure 3.

We report, in Figure 13, the power vs. frequency responses obtained with the HB and shunt rectifiers. A fit with the model of section II is also provided, showing good agreement between theory and experiments. As expected, the maximum input power obtained with the shunt-diode rectifier in the weak-coupling case is more than $13 \%$ higher than the maximum power obtained with the HB rectifier circuit. In the strongly-coupled case, the bandwidth at half maximum is approximately $5 \%$ larger with the shuntdiode circuit than with the HB rectifier circuit.
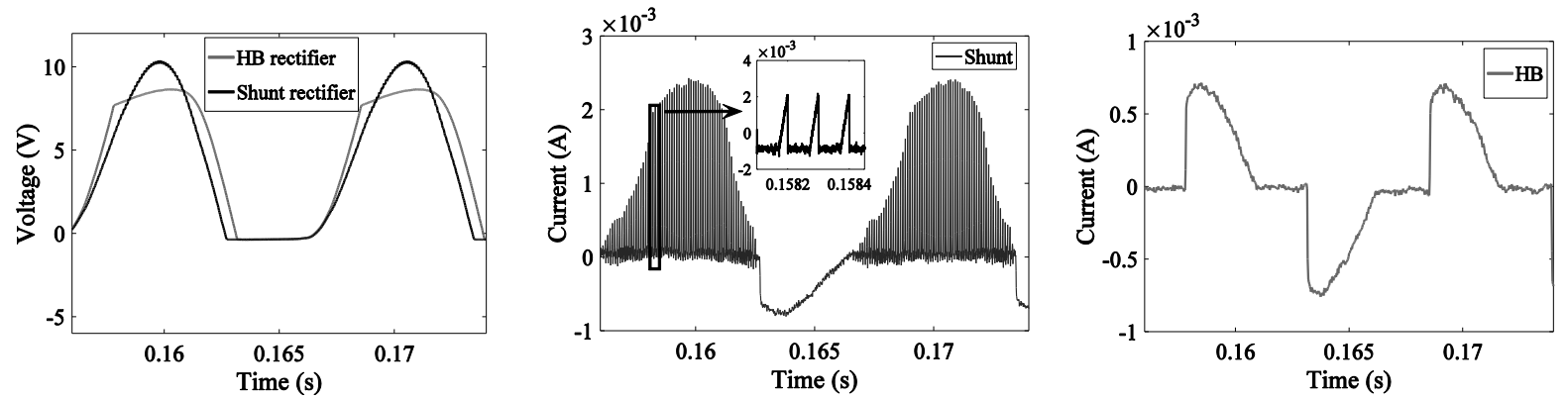

Figure 12: Typical waveforms of the piezoelectric voltages and currents for the shunt rectifier, the HB rectifier and the FB rectifier architectures (measurements). An offset has been added to the current of the HB rectifier for the sake of readability.
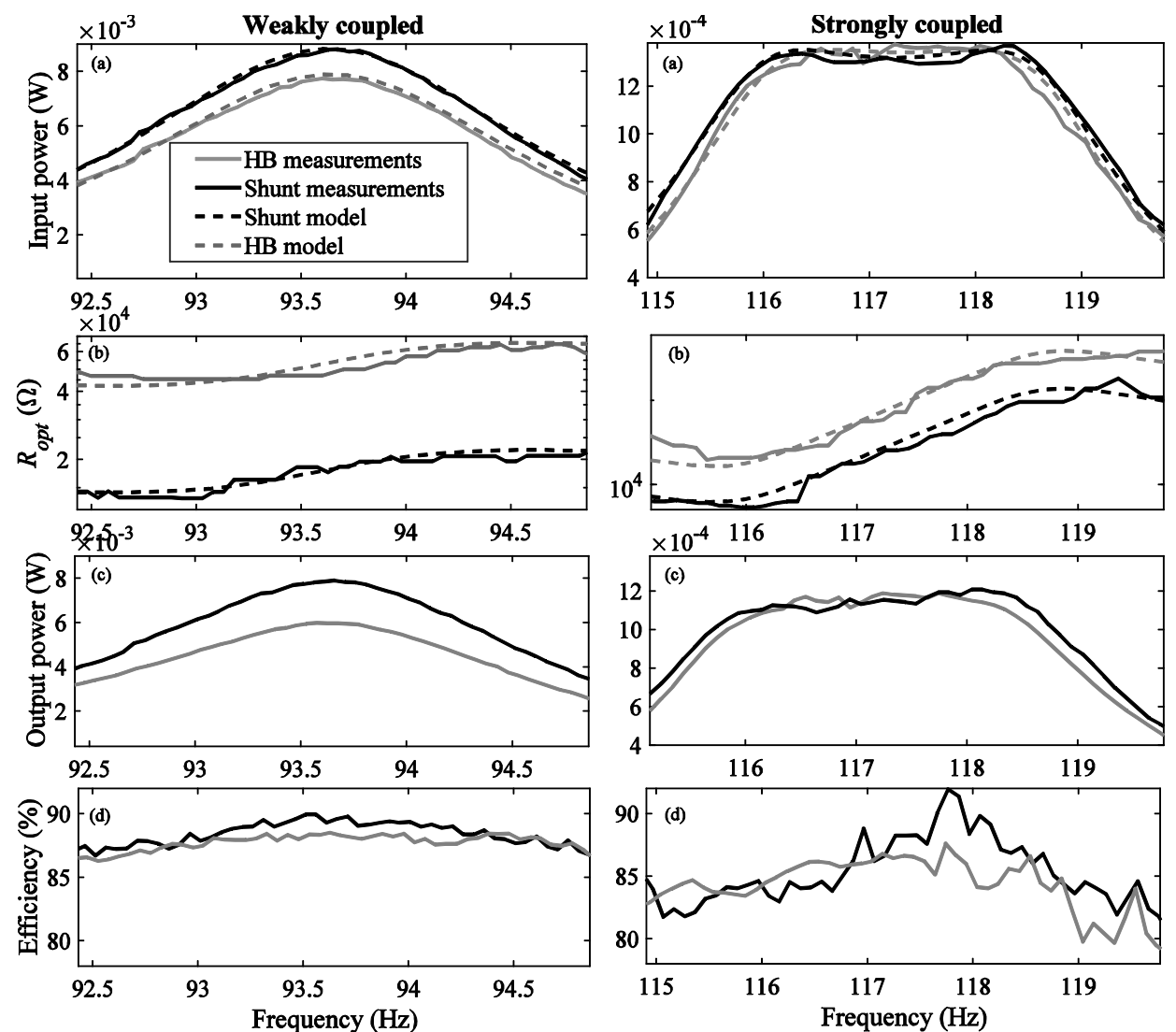

Figure 13: Measurements at high voltage (25V open-circuit voltage amplitude). Left: Weakly-coupled generator. Right: Strongly-coupled resonator. (a) Power delivered by the piezoelectric generator. (b) Optimal input impedance of the buck-boost converter. (c) Output power. (d) Electrical power efficiency. Solid lines: measurements. Dashed lines: model. 
Figure 13 also shows that, at such a high voltage level, the power efficiency of the shunt-diode architecture is similar to the power efficiency of the HB rectifier circuit because the main losses of the circuit take place in the buck-boost converter, whose efficiency can be estimated, from Figure 13, between $84 \%$ and $90 \%$ depending on the operating point. One can see that the power efficiency is slightly lower in the strongly-coupled case, where the current is larger. Hence, we believe that this (small) difference is due to resistive losses in the components (inductor and output diode of the buck-boost converter) or iron losses in the inductor.

One should keep in mind that, since the acceleration levels are set to reach similar voltage levels, they are different in the two cases. This explains why the power levels are also very different for the two resonators. In our experimental setup, $p_{\text {lim }} \simeq 21.3 \mathrm{~mW}$ for the weakly-coupled generator under $\gamma \simeq 17.7 \mathrm{~m} . \mathrm{s}^{-2}$ and $p_{\text {lim }} \simeq 1.4 \mathrm{~mW}$ for the strongly-coupled generator under $\gamma \simeq$ $4.05 \mathrm{~m} . \mathrm{s}^{-2}$. Only the strongly-coupled case reaches the maximum available input power.

\section{Additional interest of the shunt-diode rectifier at low voltage}

In subsection III.B, we have validated our model which predicts that the shunt-diode architecture extracts more power from weakly-coupled piezoelectric energy harvesters than a bridge rectifier. We have also proven that the bandwidth of the shunt rectifier system is larger than the bandwidth of a bridge rectifier circuit, especially for strongly-coupled resonators. However, in certain applications (e.g. MEMS resonators [42]), the input voltage may be very low. In such cases, the shunt-diode rectifier circuit exhibits another main advantage: it minimizes the conduction losses due to the absence of a series diode. This is especially noticeable on the electrical power efficiency at low voltages.

To illustrate this advantage, we have performed experiments at a level of acceleration for which the open-circuit voltage amplitude $u_{O C}$ at resonance is $4 \mathrm{~V}$ on the weakly-coupled resonator. The results are given in Figure 14. One can notice that, at such low level, in addition to the higher input power obtained with the shunt rectifier circuit, it also exhibits a better power efficiency. These combined advantages generate a significant gain in terms of output power $(+20 \%$ compared to the half-bridge rectifier circuit and $+43 \%$ compared to the full-bridge rectifier circuit at resonance). Contrary to the large-voltage case of Figure 13, the electrical power efficiency decreases significantly as the voltages decrease, far from the resonance frequency, because the conduction losses in the rectifier diodes become significant.
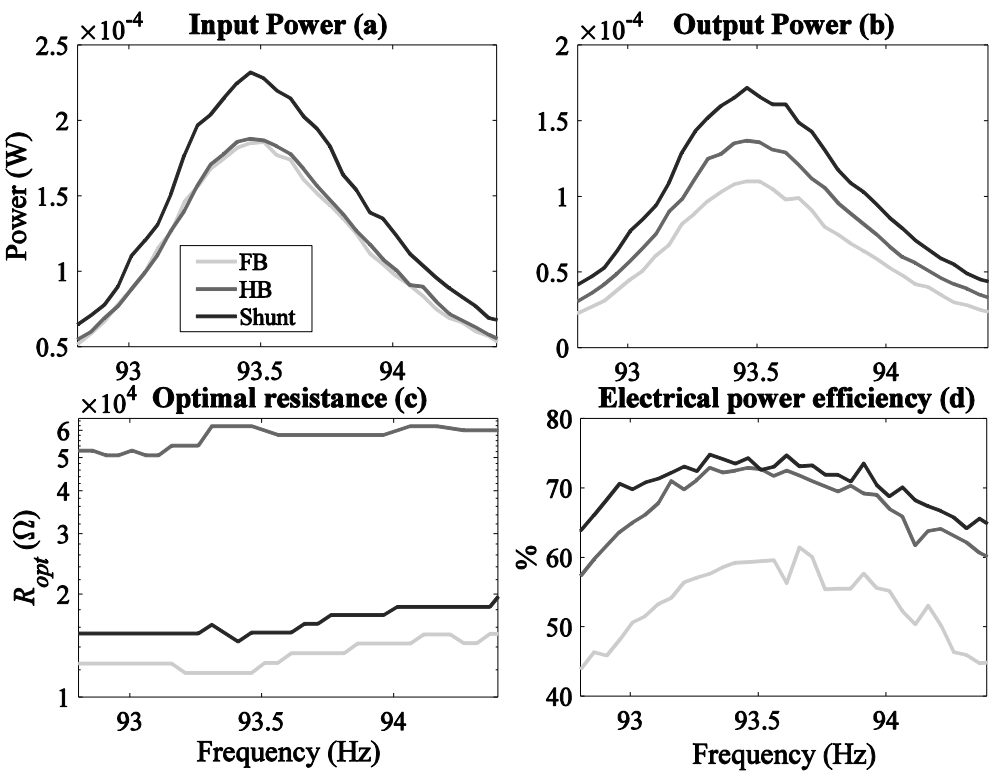

Figure 14: Measurements at low voltage for the weakly-coupled generator (4V open-circuit voltage amplitude), (a) Power delivered by the piezoelectric generator, (b) output power, (c) optimal input impedance of the buck-boost converter and (d) electrical power efficiency. Shunt-diode rectifier (dark), HB rectifier connected to smoothing capacitor (dark grey) and FB rectifier connected to smoothing capacitor (light grey).

\section{CONCLUSION}

In this paper, we have highlighted the interest of the shunt-diode rectifier for vibration energy harvesting using piezoelectric resonators. We have provided a full analytical study enabling to predict and optimize the harvester performances. The overall performance of the coupled electromechanical system has been confirmed experimentally, highlighting the interest of the shuntdiode compared to other diode-based rectifier topologies.

Our original and deepened analytical study, supported by experimental results, shows that this architecture is especially wellsuited for piezoelectric generators. For weakly-coupled generators, we have shown that the shunt-rectifier architecture brings out more power than classical HB and FB rectifiers independently of the acceleration level. Moreover, it enlarges the frequency bandwidth of the system compared to bridge rectifier circuits.

In addition to the good performances at large voltage levels, the proposed architecture is even more interesting at low piezoelectric voltages, where classical diode rectifiers degrade the power efficiency of the harvester. Further optimization of the DC-DC converter specifically designed to improve the electrical power efficiency of the shunt-diode system for piezoelectric resonators is the subject of ongoing research. 


\section{ACKNOWLEDGEMENTS}

This work was supported by the Korea Institute of Energy Technology Evaluation and Planning (KETEP) and the Ministry of Trade, Industry \& Energy (MOTIE) of the Republic of Korea (No. 20158510060040).

\section{REFERENCES}

[1] K. A. Cook-Chenault, N. Thambi, M. A. Bitetto and E. B. Hameyie, "Piezoelectric energy harvesting," Bulletin of Science, Technology and Society, vol. 28, no. 6, pp. 496-509, 2008.

[2] H. S. Kim, J.-H. Kim and J. Kim, "A Review of Piezoelectric Energy Harvesting Based on Vibration," International Journal of Precision Engineering and Manufacturing, vol. 12, no. 6, pp. 1129-1141, December 2011.

[3] Y. C. Shu and I. C. Lien, "Analysis of power output for piezoelectric energy harvesting systems," Smart Materials and Structures, vol. 15, pp. 1499-1512, 2006.

[4] J. Liang and W.-H. Liao, "Impedance Modeling and Analysis for Piezoelectric Energy Harvesting Systems," IEEE/ASME Transactions on Mechatronics, vol. 17, no. 6, pp. 1145-1157, December 2012.

[5] J. Liang and W.-H. Liao, "Improved Design and Analysis of Self-Powered Synchronized Switch Interface Circuit for Piezoelectric Energy Harvesting Systems," IEEE Transactions on Industrial Electronics, vol. 59, no. 4, pp. 1950-1960, 2011.

[6] J. Colomer-Farraron, P. Miribel-Català, A. Saiz-Vela, M. Puig-Vidal and J. Samitier, "Power-Conditioning Circuitry for a Self-Powered System Based on Micro PZT Generators in a 0.13- $\mu \mathrm{m}$ Low-Voltage Low-Power Technology," IEEE Transactions on Industrial Electronics, vol. 55, no. 9, pp. 3249-3256, September 2008.

[7] R. Dayal, S. Dwari and L. Parsa, "Design and Implementation of a Direct AC-DC Boost Converter for Low-Voltage Energy Harvesting," IEEE Transactions on Industrial Electronics, vol. 58, no. 6, pp. 2387-2396, June 2011.

[8] H. Wang, Y. Tang and A. Khaligh, "A Bridgeless Boost Rectifier for Low-Voltage Energy Harvesting Applications," IEEE Transactions on Power Electronics, vol. 28, no. 11, pp. 5206-5214, November 2013.

[9] L. Garbuio, M. Lallart, D. Guyomar, C. Richard and D. Audigier, "Mechanical Energy Harvester With Ultralow Threshold Rectification Based on SSHI Nonlinear Technique," IEEE Transactions on Industrial Electronics, vol. 56, no. 4, pp. 10481056, April 2009.

[10] A. Tabesh and L. G. Fréchette, "A Low-Power Stand-Alone Adaptive Circuit for Harvesting Energy From a Piezoelectric Micropower Generator," IEEE Transactions on Industrial Electronics, vol. 57, no. 3, pp. 840-849, March 2010.

[11] D. Guyomar, A. Badel, E. Lefeuvre and C. Richard, "Toward Energy Harvesting Using Active Materials and Conversion Improvement by Nonlinear Processing," IEEE Transactions on Ultrasonics, Ferroelectrics and Frequency Control, vol. 52, no. 4, pp. 584-595, 2005.

[12] Y. K. Ramadass and A. P. Chandrakasan, "An Efficient Piezoelectric Energy Harvesting Interface Circuit Using a Bias-Flip Rectifier and Shared Inductor," IEEE Journal of Solid-State Circuits, vol. 45, no. 1, pp. 189-204, January 2010.

[13] Y. Sun, N. H. Hieu, C.-J. Jeong and S.-G. Lee, "An Integrated High-Performance Active Rectifier for Piezoelectric Vibration Energy Harvesting Systems," IEEE Transactions on Power Electronics, vol. 27, no. 2, pp. 623-627, 2012.

[14] D. Kwon and G. A. Rincon-Mora, "A rectifier-free piezoelectric energy harvester circuit," in Circuits and Systems, 2009. ISCAS 2009. IEEE International Symposium on, Taipei, 2009.

[15] M. K. Kazimierczuk, "Analysis of Class E Zero-Voltage-Switching Rectifier," IEEE Transactions on Circuits and Systems, vol. 37, no. 6, pp. 747-755, June 1990.

[16] J. O. McSpadden, L. Fan and K. Chang, "Design and Experiments of a High-Conversion-Efficiency 5.8-GHz Rectenna," IEEE Transactions on Microwave Theory and Techniques, vol. 46, no. 12, pp. 2053-2059, December 1998.

[17] B. Strassner and K. Chang, "5.8-GHz circularly polarized rectifying antenna for wireless microwave power transmission," IEEE Transactions on Microwave Theory and Techniques, vol. 50, no. 8, pp. 1870-1876, August 2002.

[18] J. Guo, H. Zhang and X. Zhu, "Theoretical analysis of RF-DC conversion efficiency for Class-F rectifiers," IEEE Transactions on Microwave Theory and Techniques, vol. 62, no. 4, pp. 977-985, April 2014.

[19] C. Song, Y. Huang, S. Yuan and Q. Xu, "Matching network elimination in broadband rectennas for high-efficiency wireless power transfer and energy harvesting," IEEE Transactions on Industrial Electronics, vol. 65, no. 5, pp. 2305-2317, December 2016.

[20] G. Kkelis, D. C. Yates and P. D. Mitchelson, "Class-E Half-Wave Zero dv/dt Rectifiers for Inductive Power Transfer," IEEE Transactions on Power Electronics, vol. 32, no. 11, pp. 8322-8337, November 2017.

[21] A. Romani, M. Filippi and M. Tartagni, "Micropower Design of a Fully Autonomous Energy Harvesting Circuit for Arrays of Piezoelectric Transducers," IEEE Transactions of Power Electronics, vol. 29, no. 2, pp. 729-739, February 2014. 
[22] P. Gasnier, J. W. S. Boisseau, G. Despesse, C. Condemine, G. Gouvernet and J.-J. Chaillout, "An Autonomous Piezoelectric Energy Harvesting IC based on a Synchronous Multi-Shots Technique," IEEE Journal of Solid-State Circuits, vol. 49, no. 7, pp. 1561-2014, July 2014.

[23] G. K. Ottman, H. F. Hofmann and G. A. Lesieutre, "Optimized Piezoelectric Energy Harvesting Circuit Using Step-Down Converter in Discontinuous Conduction Mode," IEEE Transactions on Power Electronics, vol. 18, no. 2, pp. 696-703, March 2003.

[24] G. K. Ottman, H. F. Hofmann, A. C. Bhatt and G. A. Lesieutre, "Adaptive piezoelectric energy harvesting circuit for wireless remote power supply," IEEE Transactions on Power Electronics, vol. 17, no. 5, pp. 669-676, September 2002.

[25] S. Arimoto, H.-Y. Han, C. C. Cheah and S. Kawamura, "Extension of impedance matching to nonlinear dynamics of robotic tasks," Systems \& Control Letters, vol. 36, pp. 109-119, 1999.

[26] E. Lefeuvre, A. Badel, A. Brenes, S. Seok, M. Woytasik and C.-S. Yoo3, "Analysis of piezoelectric energy harvesting system with tunable SECE interface," Smart Materials and Structures, vol. 26, no. 3, February 2017.

[27] A. Badel and E. Lefeuvre, "Nonlinear Conditioning Circuits for Piezoelectric Energy Harvesters," in Nonlinearity in Energy Harvesting Systems, E. Blokhina, A. E. Aroudi, E. Alarcon and D. Galayko, Eds., Cham, Springer, 2016, pp. 353-357.

[28] A.Banerjee, B.Bhattachary and A.K.Mallik, "Large deflection of cantilever beams with geometric non-linearity: Analytical and numerical approaches," International Journal of Non-Linear Mechanics, vol. 43, no. 5, pp. 366-376, 2007.

[29] N. G. Elvin and A. A. Elvin, "Large deflection effects in flexible energy harvesters," Journal of Intelligent Material Systems and Structures, vol. 23, no. 13, pp. 1475-1484, 2012.

[30] Y. Liao and H. A. Sodano, "Model of a single mode energy harvester and properties for optimal power generation," Smart Materials and Structures, vol. 17, p. 065026, November 2008.

[31] D. W. Hart, Introduction to Power Electronics, Upper Saddle River: Prentice Hall, 1997.

[32] N. Kong, D. S. Ha, A. Erturk and D. J. Inman, "Resistive Impedance Matching Circuit for Piezoelectric Energy Harvesting," Journal of Intelligent Material Systems and Structures, vol. 21, pp. 1293-1302, 2010.

[33] R. D'hulst, T. Sterken, R. Puers, G. Deconinck and J. Driesen, "Power Processing Circuits for Piezoelectric Vibration-Based Energy Harvesters," IEEE Transactions on Industrial Electronics, vol. 57, no. 12, pp. 4170-4177, 2010.

[34] E. Lefeuvre, D. Audigier, C. Richard and D. Guyomar, "Buck-Boost Converter for Sensorless Power Optimization of Piezoelectric Energy Harvester," IEEE Transactions on Power Electronics, vol. 22, no. 5, pp. 2018-2025, September 2007.

[35] S. Pryia, "Criterion for Material Selection in Design of Bulk Piezoelectric Energy Harvesters," IEEE Transactions on ultrasonics, ferroelectrics and frequency control, vol. 57, no. 12, pp. 2610-2612, 2010.

[36] P.-H. Hsieh, C.-H. Chen and H.-C. Chen, "Improving the Scavenged Power of Nonlinear Piezoelectric Energy Harvesting Interface at Off-Resonance by Introducing Switching Delay," IEEE Transactions on Power Electronics, vol. 30, no. 6, pp. 3142-3155, 2015.

[37] S. L. F. Boussaid., "A highly efficient P-SSHI rectifier for piezoelectric energy harvesting," IEEE Transactions on Power Electronics, vol. 30, no. 10, pp. 5364 - 5369, 2015.

[38] D. A. Sanchez, J. Leicht, E. Jodka, E. Fazel and Y. Manoli, "A 4 $\mu$ W-to-1mW parallel-SSHI rectifier for piezoelectric energy harvesting of periodic and shock excitations with inductor sharing, cold start-up and up to $681 \%$ power extraction improvement," in IEEE International Solid-State Circuits Conference, San Francisco, 2016.

[39] I. C. Lien, Y. C. Shu, W. J. Wu, S. M. Shiu and H. C. Lin, "Revisit of series-SSHI with comparisons to other interfacing circuits in piezoelectric energy harvesting," Smart Materials and Structures, vol. 19, 2010.

[40] K. Li, "Structural vibration damping with synchronized energy transfer between piezoelectric patches," INSA, Lyon, 2011.

[41] M. Lallart, Y.-C. Wu and D. Guyomar, "Switching Delay Effects on Nonlinear Piezoelectric Energy Harvesting Techniques," IEEE Transactions on Industrial Electronics, vol. 59, no. 1, pp. 464-472, 2011.

[42] R. Sriramdas and R. Pratap, "Scaling and Performance Analysis of MEMS Piezoelectric Energy Harvesters," Journal of Microelectromechanical Systems, vol. 26, no. 3, pp. 679-690, 2017. 